\title{
Fault Detection in Fiber Optic Communication Cable by Coherent Anti-Stokes Raman Scattering using Superconducting Nanowire Single-Photon Detector
}

\author{
Annepu Venkata Naga Vamsi ${ }^{*}$ and Annepu Bhujanga Rao ${ }^{2}$ \\ 'Department of Electronics and Instrumentation, Gitam University, Visakhapatnam - 530045, Andhra Pradesh, \\ India; vamsi9441105975@gmail.com \\ 2Department of Instrument Technology, Andhra University, Visakhapatnam - 530003, \\ Andhra Pradesh, India; dr_abrao@yahoo.co.in
}

\begin{abstract}
Objective: In this paper, the author has used the coherent anti-stokes Raman scattering and the time correlated single photon count technique to locate the fault position and fault in fiber optic communication cable. Method/Statistical Analysis: The coherent anti-stokes Raman scattering where used to measure the single photon count and the counts are correlated with time to measure the position of the fault in fiber optical cable. The proposed method is experimented on a 4 meter length fiber optic cable which is under test. The experiment is performed by considering prefixed faults at different length of the fiber optic cable under test. The author has done one of its kinds of experimentation to measure the faults and fault position in the fiber optical cables, lay underneath the ground. Findings: The experimented results have shown a clear cut edge with the technique that the photon count rate is decreased at the fault position of the optical fiber cable under test. The sharp increase of counts after the fault position and sharp decrease of counts before the fault position strongly supports the proposed method for fault finding in fiber optic cable. Application/Improvement: The proposed method is much more accurate in finding faults which is not possible by the pervious technology or devices. The proposed method can be applied to lengthy fiber optic cables by selecting high range pump lasers and advanced filters.
\end{abstract}

Keywords: Coherent Raman Scatterings, Detection Efficiency, Photon Count Rate, Superconducting Nanowire Single -Photon Detector ,

\section{Introduction}

Fiber optic communication has revolutionized the telecommunication industry. Using fiber optic cable, optical communications have enabled telecommunication links to be made over longer distances and with much low levels of loss in the transmission medium. Since the earliest days of telecommunications there has been an ever increasing need to transmit high data even faster. In the earlier time, single line wires were used. Which gave way to coaxial cables and later on the invention of laser, the fiber optic communication with its advantages marked the beginning of high rate date transmission and thus the fiber optic is a bigger building block in the communication sector. With its higher bandwidth capabilities and low attenuation characteristics makes it ideal for megabytes to gigabit transmission and even more.

With the advancement in the optical sources and photo detectors which are capable of operating at 1500 $\mathrm{nm}$ changed the transmission wavelength from $800 \mathrm{~nm}$. As one can use both multimode and single mode fibers in local area networks, where bit rate ranges in the order 10 $100 \mathrm{Mb} / \mathrm{s}$ over longer distances. By the high quality lasers and high quality receivers allowed single wavelength transmission rates of around $10-20 \mathrm{~Gb} / \mathrm{s}$.

In the 1970s,fiber-optic communication systems have

\footnotetext{
${ }^{*}$ Author for correspondence
} 
revolutionized the telecommunication industry, Because of its advantage over electrical transmission, optical fiber have largely replaced copper wire communication in core networks in the well developed countries. In the mid1990's, a combination of erbium-doped fiber amplifiers (EDFAs) and wavelength-division multiplexing (WDM) were used to boost fiber capacity to higher levels and also to increase the transmission distance. A major consideration in these super high links is to ensure that there is appropriate link, so that alternate paths are required in case of disruptions in communications caused by cable ruptures (e.g., caused by errant digging from a backhoe) or a major cuts or bends in the communication cables which are laid underground, such disruption could otherwise have an adverse effect on the end users.

While communicating at longer distances, where there is a probability of damage caused either externally or internally during installation or after installation, this damage can lead to different losses and thereby causing the transmission problems. In this paper we have discussed about a method by which we can find the faults in the cable, by using the coherent anti-stokes Raman scatterings along with the superconducting nano wire single -photon detectors (SNSPDs).

The optical fiber based sensors are the most suitable devices than the multiplexed sensors as we can replace many individual sensors which simplify sensor maintenance and installations. The major classes of fiber sensor are the optical frequency domain reflectometry (OFDR) and optical time-domain reflectometry (OTDR). The OFDR system ranges is limited to tens of meters and are based on interferometric method, thus a greater level of care required to avoid measurement related problems associated with the polarization and vibration. The OTDR measurements systems typically consist of a light source and high sensitivity receiver, a data - acquisition module, information storage information -storage unit. The OTDR is basically optical radar. It operates by periodically launching narrow laser pulses into one end of a optical fiber under test by using a directional coupler or a beam splitter. The properties of the optical fiber link are then determined by analyzing the amplitude and temporal characteristics of the waveform of the backscattered lightt. J.P. Dakin et al were the first to measure the distributed temperature from Raman scatterings'. Bolognini et al. measured distance range greater than $40 \mathrm{~km}$ in single-mode dispersion shifted fiber, using optical amplification In this Paper we used the coherent anti-stokes Raman scattering along with the single -photon detectors (SNSPDs) to measure the intensity at the receiver end of the optical fiber. We also calculated the time taken for the anti-stokes photons to reach the detector from the fiber under test (FUT) which is used to find the faulty position in the test fiber, as show in the figurel. By the time-correlated single-photon counting technique we have analyzed the time delays between the launch of a laser pulse to the detection of back scattered photons at the receiving detectors. Thus we were able to plot a graph as shown in the Figure 3.

\section{Theoretical Modeling of Detector Counts}

The author modeled the detector count rate derived from the Raman scatterings as coherent stokes Raman scattering and coherent anti-stokes Raman scattering] as follows:

$l_{A}=\eta_{\mathrm{A}} \Delta V_{A} P_{0} L\left|g_{R}, A\right| D_{c} \Omega$

Where $l_{A}$ is the number of backscattered photons per second from anti-stokes, $\eta_{A}$ is a product of the detection efficiency (DE) of the SNSPDs and the transmission coefficient of the filter chains, $\Delta V_{A}$ is the bandwidth of the filters applied to the Stokes and anti-Stokes channels, $\mathrm{P} 0$ is the maximun peak pump power, $\mathrm{L}$ is the length od the fiber under test, $g_{R}, A$ is the Raman gain coefficient, $D_{c}$ is the pump signal duty cycle, $\Omega$ is the radial frequency detuning between the pump signal and the wavelength of the Stokes or anti-Stokes.

$l_{s}=\eta_{s} \Delta V_{s} P_{0} L\left|g_{R}, S\right| D_{c} \Omega$

Where, the subscript $S$ represents stokes in the above equation ${ }^{2}$.

As the author approximate the Raman gain coefficient and detuning $\Omega$ to be independent of frequency over stokes and anti-stokes filter bandwidths.

Thus the ratio of stokes to anti-stokes photon count rate is given by equation (3) from the quantum mechanics domain as,

$\frac{l_{A}}{l_{S}}=\left(\frac{\lambda_{S}}{\lambda_{A}}\right) I^{-4} \exp \left(-\frac{\hbar \omega_{M}}{K_{B}}\right)$

Where $l_{A}$ and $l_{S}$ are the anti-stokes and stokes count rate, while $\frac{\lambda_{s}}{\lambda_{A}}$ is the detector efficiency. Thus from the 
above equations, we were able to calculate the photon count rate and the detection efficiency at the receiver end of the fiber under test.

\section{Experiment Set Up}

The experimental measurement set up is as shown in Figure 1., where the optic source is a laser with a wavelength of 1550 which has frequency repletion of $45 \mathrm{MHz}$. For the experimentation, we have used a lowloss single -mode fiber cable which has a core diameter of $10 \mu \mathrm{m}$ and a cladding diameter of $125 \mu \mathrm{m}$ (SM9805.8-125) with a wavelength of 980-1550 nm. For better reduction of the unwanted wavelengths we have filtered the laser light to a $1 \mathrm{~nm}$ line width at a wavelength of $1533.47 \mathrm{~nm}$ in international telecommunicaion union channel no.55. The pump laser is passed through the pumped filter and is amplified with a fiber amplifier (FA).The amplified signal is again passed though another filter to remove any anti stokes echoes from the pump laser, which can create unwanted counts at the photon detectors. As we have used filtering at the pre and post of the EDFA, thus we have successfully rejected 20 to $28 \mathrm{~dB}$ of undesirable wavelengths. We now injected the pump laser light signal into our test fiber cable, for experimentation. In this experiment, the author used a single - mode fiber as a testing fiber. The length of the test fiber is limited to 4 meters length due to low repetition rate of the pump laser. We expect that this proposed technique can easily be applied to lengthy fiber optic cables by selecting suitable modulator applied to the pump laser. The output signal from the test fiber is passed through the band splitters to separate the Raman backscattered photons into two bands. The S-band channels from (1450-1490nm) and an L-band channels from (1530-1570 nm). Once we have the bands separated, the wavelengths with their counts are detected by the superconducting nano wire single photon detectors. By the time-interval analyzer (TIA), we are able to create histograms/plot for the time delays between laser clock electrical pulse to the pulses generated by superconducting nanowire single photon detector.

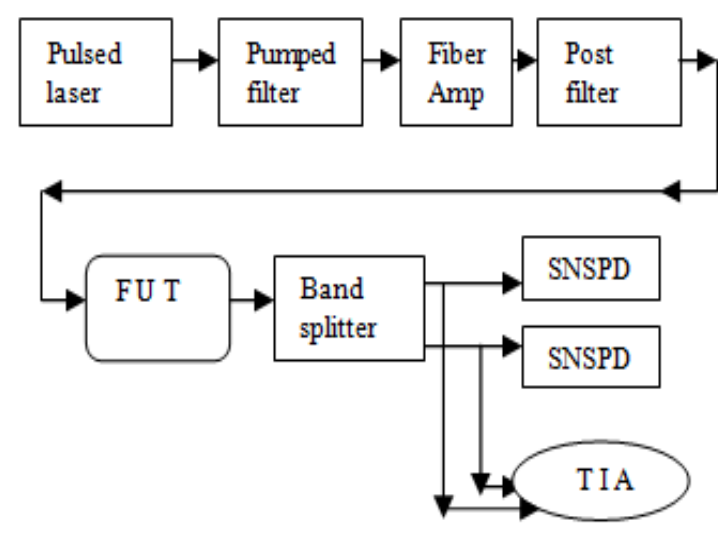

Figure 1. Block Diagram of the Experimental Set Up for the Measurement of Photon Count Rate, Time Interval Analyzer for Fault Detection in Fiber Cable.

The gain coefficient in fibers increases with detuning, but it is quite small, hence neglected. The SNSPDs used in this paper had relatively low detection efficiency (Des), in addition, we have to account the losses of filters used in the experimental set up. As the pump power is limited in our experiment, therefore, we are left with the option of increasing the bandwidth of stokes and anti-stokes filters so as to increase the count rate. The filter range used for stokes is of $1550 \mathrm{~nm}-1610 \mathrm{~nm}$ and anti-stokes filter range is of $1450 \mathrm{~nm}-1490 \mathrm{~nm}$.

\section{Experiment Results}

As the histogram counts per bin due to the coherent antistokes Raman scattering is determined as follow:

$N_{\downarrow} u[m]=l_{u}[m] t_{\text {int }}$

$\approx \eta_{u} \Delta u P_{o} L\left|g_{R}, u\right| D_{c} \Omega$

$m=\frac{x}{L_{b i n}}$

Where $t_{\text {int }}$ is the integration period, and Lbin $=\mathrm{c}$ tbin $/ 2$ nfiber is the equivalent length of the time bins (tbin).

The bin variable $m=\frac{x}{L}$ represents the fault position of the fiber under test in the equation ${ }^{4}$ and $^{5}$. From the comparison of the Eq. (1\&2) we get the Raman scatterings in terms of photons per second, while the 
equation ${ }^{4}$ gives the total number of photons counts. Thus with this theoretical modeling we have found the fault position by experimentally setting up the test fiber under different bending positions (in meters) and allowing the pump signal to pass through the bend positions of the fiber under test as shown in the figure 2. where the time taken by the pump signal to receive at the receiver end is noted and analyzed by the time interval analyzer (TIA) and which finally determine the position of the fault (bend) in the fiber under test.

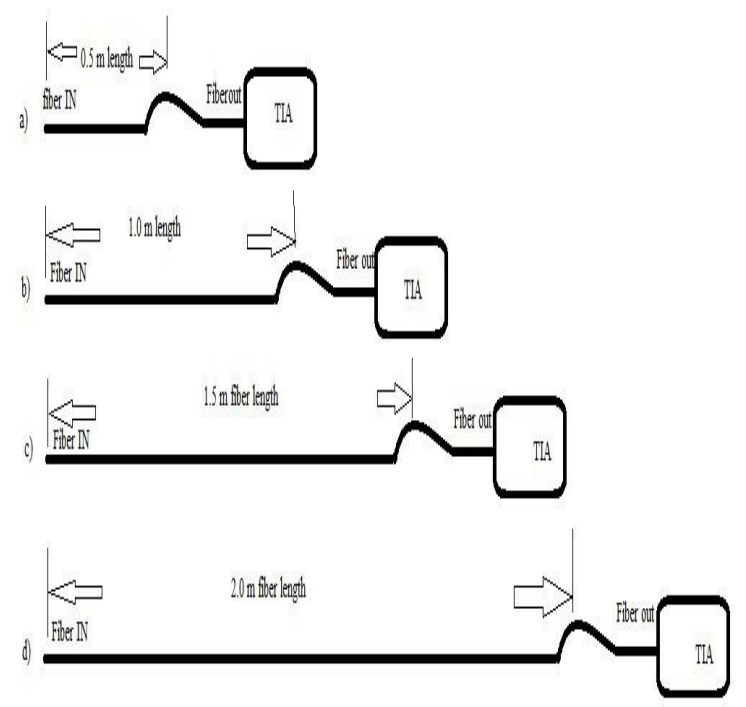

Figure 2. Test Fiber and the Bend (fault) Position at (a) 0.5 meters (b) 1.0 meters (c) 1.5 meters (d) 2.0 meters up to 3.0 meters and with the TIA (Time Interval Analyzer).

We have done the experiment for different lengths of the bends in the optical fiber cable starting from 0.5 meters, 1.0 meters, 1.5 meters, 2.0 meters, 2.5 meters, 3.0 meters respectively and we have observed that the photon count rate and the time taken by the signal to reach the receiver are been reduced and this is due to the bends in the optical fiber cable which is intentionally bended for the experimentation purpose. The proposed method in this paper has successfully achieved the objective of finding the fault location underneath the ground. The observed time interval by the time interval analyzer with respect to the position is shown in the figure 3 .

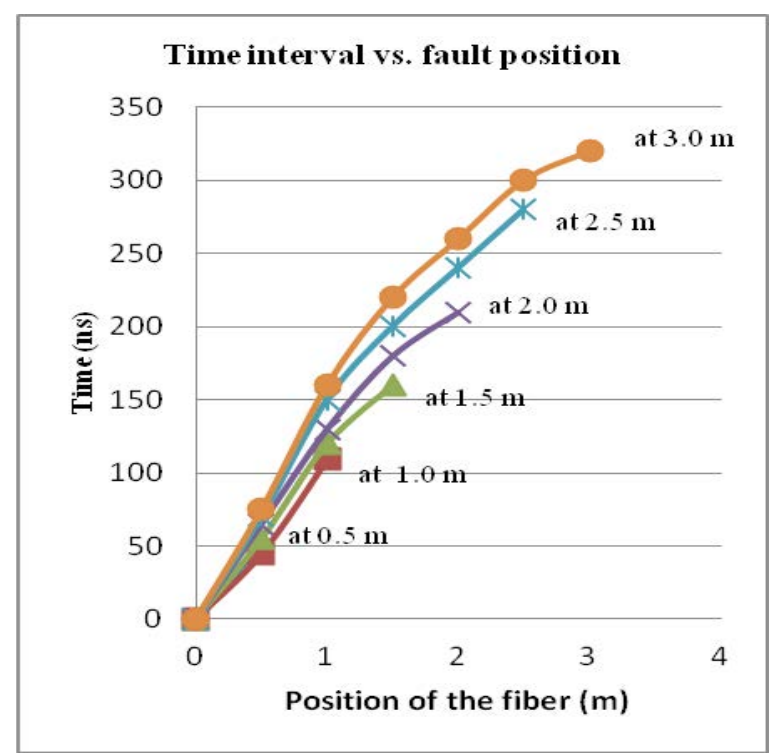

Figure 3. Results of the TIA (Time Interval Analyzer) with the Different Fault Positions from 0.5 m, 1.0 m, 1.5 $\mathrm{m}, 2.0 \mathrm{~m}, 2.5 \mathrm{~m}$, and $3.0 \mathrm{~m}$.

We have plotted the measured photon count rate vs. Position of the fiber in meters. which clearly shows that the photon count rate is reduced at the fault positions and is increased before and after in the test fiber under test. The figure 4 , the plot shows that at sharp 0.5 meters the photon count is reduced from 460 counts to 350 counts, which clearly indicates the bend in the fiber.

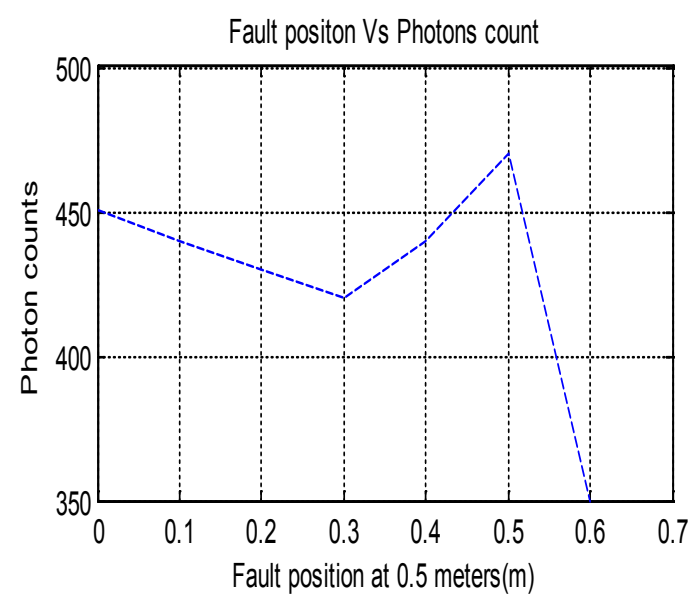

Figure 4. Plot for Fault Position in the Fiber (meters) vs. the Photon Count Rate at $0.5 \mathrm{~m}$. The Count Rate Decrease at Fault Position. 


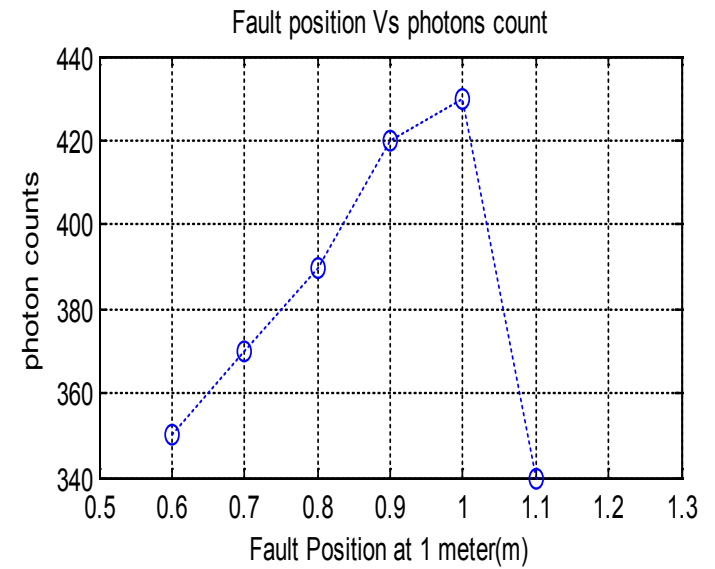

Figure 5. Plot for Fault Position in the Fiber (meters) vs. the Photon Count Rate at $1 \mathrm{~m}$. The Count Rate Decrease at Fault Position.

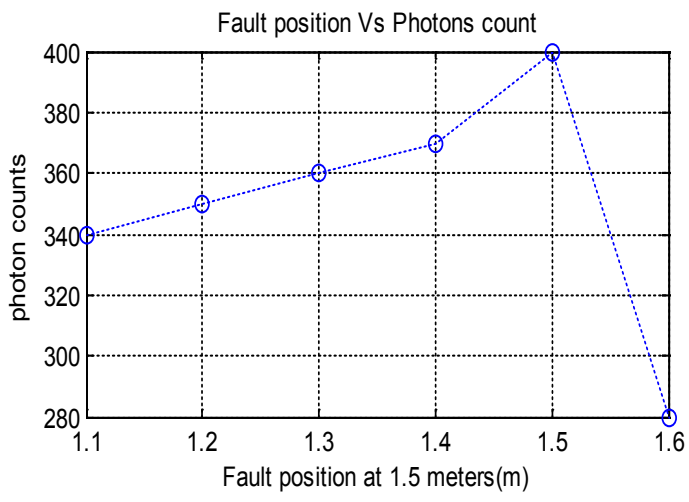

Figure 6. Plot for Fault Position in the Fiber (meters) vs. the Photon Count Rate at $1.5 \mathrm{~m}$. The Count Rate Decrease at Fault Position.

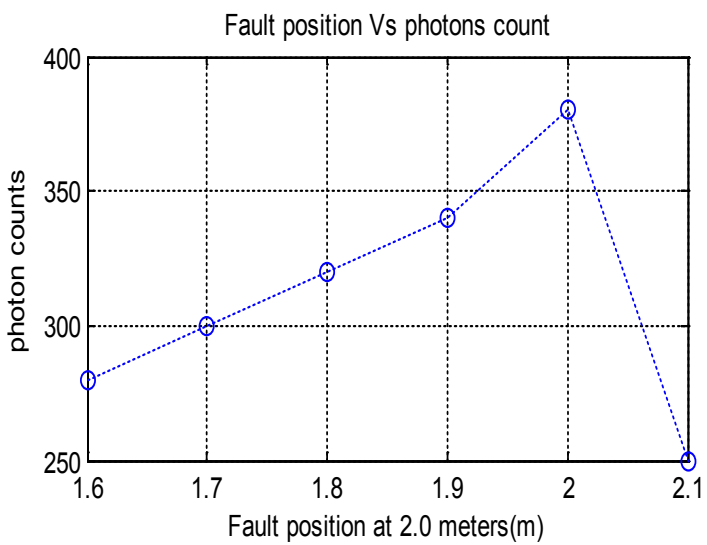

Figure 7. Plot for Fault Position in the Fiber (meters) vs. the Photon Count Rate at $2.0 \mathrm{~m}$. The Count Rate Decrease at Fault Position.

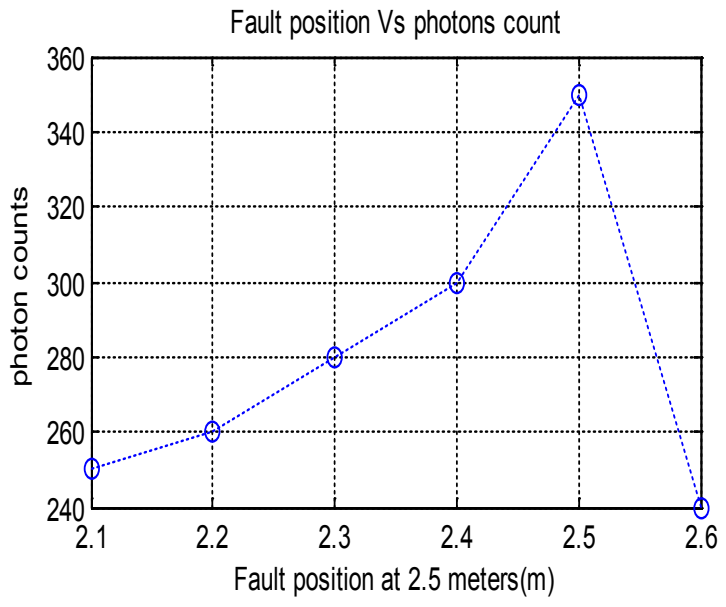

Figure 8. Plot for Fault Position in the Fiber (meters) vs. the Photon Count Rate at $2.5 \mathrm{~m}$. The Count Rate Decrease at Fault Position.

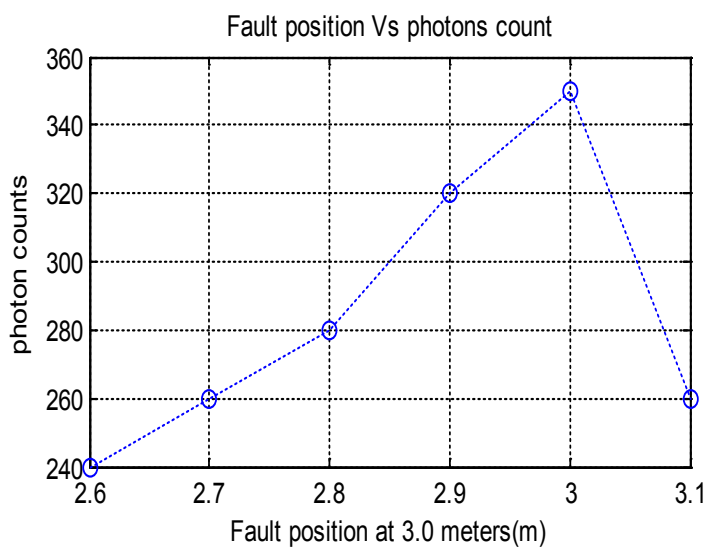

Figure 9. Plot for Fault Position in the Fiber (m) vs. the Photon Count Rate at $3.0 \mathrm{~m}$. The Count Rate Decrease at Fault Position.

The same results have been spotted for the different fault positions of the fiber which are shown in the Figure 5, Figure 6, Figure 7, Figure 8 and Figure 9 respectively. So from the above results we can clearly state the position and the fault in the fiber with the proposed method.

\section{Conclusion}

With the above results, we have demonstrated a new method of finding faults in a fiber optic communication cables which are laid underneath the ground layer. By the above experimentation we have achieved up to 3 meters length test under fiber and can be applied to a 
longer length also. The sensing system proved to be most accurate for finding the exact location of the fault.

Finally, the precise location of fault in the 3 meter long section of the fiber optic cable under test was demonstrated and results obtained with the proposed method is achieved successfully. The accuracy and performance of finding the faults in the optical communication cables of longer length can be done by improving the pump power, lower loss filters and proper coupling of fiber to the detectors.

\section{References}

1. Gerd keiser. Optical Fiber Communications. McGraw-Hill international editions; 2000.

2. Dakin JP,Pratt DJ. Distributed Optical Fibre Raman Temperature Sensor using a Semiconductor Light Source and Detector. Electronics Letters.1985; 21(13): 569-70.

3. Bolognini G, Park J, Soto MA, Park N, Pasquale FD. Anal- ysis of Distributed Temperature Sensing based on Raman Scattering using OTDR Coding and Discrete Raman Amplification. Measurement Science and Technology. 2007; 18(10): 3211-18.

4. Feced R, Farhadiroushan M,. Handerek VA, Rogers A J. Advances in High Resolution Distributed Temperature Sensing using the Time-Correlated Single Photon Counting Technique. IEE Proc.-Optoelectron. 1997; 144(3): 183--8.

5. Stierlin R, Ricka J, Zysset B, Bättig R, Weber HP, Binkert T, Borer WJ. Distributed Fiber-Optic Temperature Sensor using Single Photon Counting Detection. Applied Optics. 1987; 26(8): 1368-70..

6. Rahul Kumar Gujral, Damodaran V, Kaustab Ghosh. A Theoretical Analysis of the Dark Current in Quantum Dot Infrared Photodetector using Non- Equilibrium Green's Function Model. Indian Journal of Science and Technology. 2016 Sep; 9(36): DOI: 10.17485/ijst/2016/v9i36/102908

7. Venkata Naga Vamsi A. Coherent Anti-Stokes and Coherent Stokes in Raman Scattering by Superconducting Nanowire Single -Photon Detector for Temperature Measurement. Sensors and Transducers.2016;196(1):24-9. 